\title{
On the Importance of ATP Energy in Biology with Regard to Viruses
}

\author{
Fritz Lewertoff \\ Service de Recherche Pédagogique, Québec, Canada \\ Email: f.lewer@sympatico.ca
}

How to cite this paper: Lewertoff, F. (2021) On the Importance of ATP Energy in Biology with Regard to Viruses. Open Journal of Biophysics, 11, 225-232. https://doi.org/10.4236/ojbiphy.2021.113008

Received: March 17, 2021

Accepted: May 24, 2021

Published: May 27, 2021

Copyright $\odot 2021$ by author(s) and Scientific Research Publishing Inc. This work is licensed under the Creative Commons Attribution International License (CC BY 4.0).

http://creativecommons.org/licenses/by/4.0/ (c) (i) Open Access

\begin{abstract}
Even today in light of the pandemic spread of the corona virus COVID 19, the debate amongst biologists which concerns whether viruses are alive or not still remains unresolved. This, however, revolves around the argument that viruses cannot produce their own adenosine triphosphate molecule (ATP) through metabolism and, therefore, must rely on other living microorganisms that can produce it in order to access the energy that ATP provides upon hydrolysis. It is mainly on account of this distinction that viruses are relegated to an ill-defined area that separates living things from nonliving things. Nevertheless, apparently viruses possess an inherent capacity that enables them to cross this invisible dividing line in order to link up with the ATP molecule through which they sustain their activities. The following presents a review of these issues with regard to microbiology, biochemistry and physics.
\end{abstract}

\section{Keywords}

ATP, Virus, Autonomy, Collaboration, Lineage Formation, Living, Non-Living, Metabolic Whole

\section{Introduction}

In "Varieties of Living Things: Life at the Intersection of Lineage and Metabolism" [1], hereafter referred to as "the essay", John Dupré and Maureen A. O'Malley address three fundamental questions: 1) What does it mean for an entity to be living? 2) What is the role of inter-organismic collaboration in evolution? 3) What is a biological individual? And, apparently in light of these questions, their central argument is that "life arises when lineage-forming entities collaborate in metabolism". However, their analysis left some questions unanswered about microorganisms and particularly with respect to the biological status of viruses. It seemed that some relevant information was missing that would 
more explicitly tie the central argument to the initial fundamental questions. In other words, although the Dupré \& O’Malley essay [1] covered biological and evolutionary issues quite satisfyingly, more detailed explanations seem to be required with regard to what metabolism essentially entails in biochemical terms.

For example, the important role that the ATP molecule plays in metabolism was not mentioned even once by Dupré \& O’Malley [1] although its discovery dates back to 1929 [2]. Moreover, since ATP is the energy currency for life, it can be argued, in order to establish a connection between the above central argument and fundamental question, that the important role played by ATP cannot be circumvented. Fundamentally, there are two ways that a microorganism can be considered, either (a) the organism has the apparatus for metabolism, which means that it has the property or capacity to produce and use ATP for its energy needs, or (b) the organism does not have the apparatus to produce ATP but, nevertheless, has the capacity or capability to partake in existing metabolic processes to satisfy or derive its energy needs. In what follows we will refer to (a) and (b) in light of the fundamental questions (1), (2) and (3) with particular emphasis on viruses.

\section{What Does It Mean for an Entity to Be Living? (1)}

In his book "What is Life?" [3] under the subheading of "Living Matter Evades the decay to Equilibrium", Ervin Schrödinger asks "What is the characteristic feature of life? When is a piece of matter said to be alive?" He then answers his own questions saying it is alive "when it goes on doing something, moving, exchanging material with its environment for a much longer period than we would expect of an inanimate piece of matter to keep going under similar circumstances" ([3], p. 24). This argument allows that there is an observable or experimentally detectable dividing line between which things can be considered as alive and which things cannot be considered alive. Besides If we now accept the essay's argument that "metabolism, the transformative biochemical reactions that life processes, is a collaborative affair" ([1], p. 2), then it may be considered that (1) has been answered. But (2) must still be considered in context with respect to (a) and (b) in order to establish what role ATP plays in any collaborative endeavors.

\section{What Is the Role of Inter-Organismic Collaboration? (2)}

In his article "ATP: The Perfect Energy Currency for the CelP" [4], Jerry Bergman gives us an excellent account of the role that adenosine phosphates, namely, adenosine monophosphate (AMP), adenosine diphosphate (ADP\}, and adenosine triphosphate (ATP) play in biochemical reactions. Here are several points from Bergman's article that are relevant to the present discussion.

- The ubiquitous ATP molecule is used to build complex molecules, contract muscles, generate electricity in nerves, and light fireflies. All fuel sources of nature, all food-stuff of living things, produce ATP, which in turn powers 
virtually every activity of the cell and organism ([4], p. 1).

- Energy is usually liberated from the ATP molecule to do work in the cell by a reaction that removes one of the phosphate-oxygen groups to form ADP. When the ATP converts to ADP, the ATP is said to be spent. The ADP is then usually immediately recycled in the mitochondria where it is recharged back into ATP ([4], p. 2).

- The enormous amount of activity that occurs inside each of the approximately one hundred trillion human cells are shown by the fact that at any instant, each cell contains about one billion ATP molecules. This amount is sufficient for the cells needs for only a few minutes and must be rapidly recycled ([4], p. 2).

- The total human body content of ATP is only about 50 grams, which must be constantly recycled daily. The ultimate source of energy for constructing ATP is food. ATP is simply the carrier and regulation-storage unit of energy ([4], p. 3).

- ATP is used for many cell functions including transport work and moving substances across cell membranes ([4], p. 4).

- ATP manufacture requires several cell processes including fermentation, respiration, and photosynthesis. Most commonly the cells use ADP as a precursor molecule and then add a phosphorus. In eukaryotes this can occur either in the soluble portion of the cytoplasm (cytosol) or in special energy-producing structures called mitochondria. Charging ADP to ATP in the mitochondria is a process called phosphorylation. This process occurs in specially constructed chambers located in the mitochondrion's inner membranes ([4], p. 4). A more extensive description of these processes involving the linkage of the budding of enveloped viruses with the energy of ATP can be found in [5].

- Although ATP contains the amount of energy necessary for most reactions, at times more energy is required. The solution is for ATP to release two phosphates instead of one, producing one AMP plus a chain of two phosphates called a pyrophosphate. How AMP is built up to ATP again illustrates both the precision and the complexity of the cell energy system. The enzymes used in glycolysis, the citric acid cycle, and the electron transport system, are all precise to a degree that they will replace only a single phosphate. They cannot add two new phosphates to an AMP molecule to form ATP. The solution is an intricate enzyme called adenylate kinase, which transfers a single phosphate from an ATP to the AMP, producing two ADP molecules. The two ADP molecules can then enter the normal Krebs cycle designed to convert ADP into ATP ([4], p. 6). Figure 1, provided by Jerry Bergman [4], shows the relationship that ATP maintains with ADP and AMP.

In light of the above, inter-organismic collaboration between macromolecules and cell organelles, in particular between ATP and mitochondria, cannot be doubted to increase metabolism. Also (a), that a microorganism can produce 


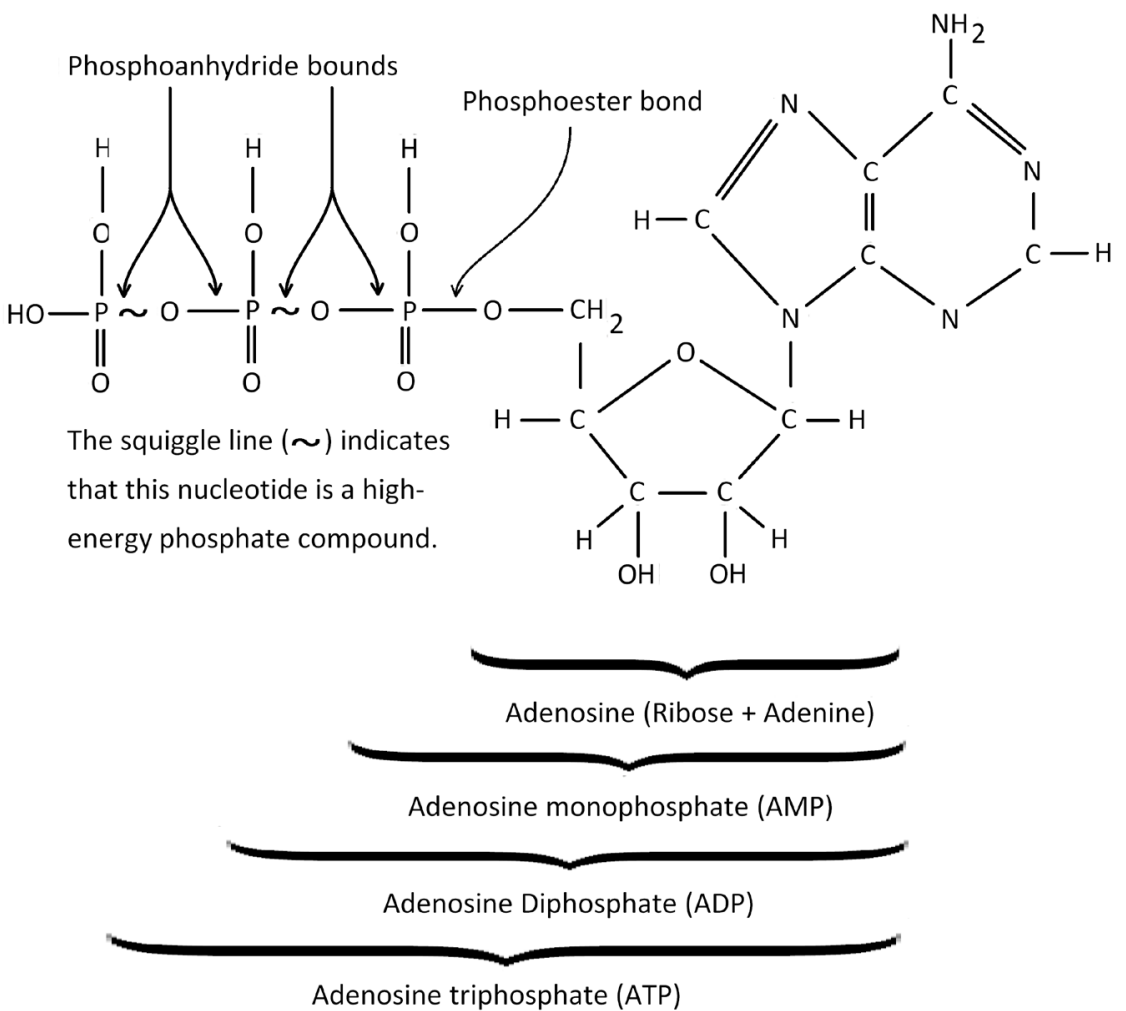

Figure 1. The two-dimensional stick model of the adenosine phosphate family of molecules, showing the atoms and bonds arrangement.

and use ATP, is true. However, how this relates to evolution and (b), how a microorganism can use ATP without being able to produce it, is unknown. Let us now move on to (3) to provide what remains to be said to satisfy (2).

\section{What Is a Biological Individual? (3)}

The essay [1] indicates that numerous keywords are of particular importance here, namely: Autonomy, Collaboration, Lineage formation, Living, Metabolic whole, None-living ([1], p. 1). Here we will briefly consider how these keywords relate to a biological individual within the context of the essay.

\subsection{Autonomy}

The essay holds that it is entirely reasonable to view autonomy as centrally exhibited in collaboration rather than ragged independence. If this kind of autonomy is required in order to count as living viruses, which were discovered for the first time at the turn of the $19^{\text {th }}$ century [6], count not only as living matter, but as full-blown entities able to interact with the cell's metabolic capacities ([1], p. 15).

\subsection{Collaboration}

Although biological theorists assign importance to competition, giving collaboration proper emphasis could provide important insight into the nature of evolutionary processes because it affects how we conceptualize the entities and ac- 
tivities central to evolution ([1], p. 14). Many ecological examples of collaboration exist which are at least as compelling as those that highlight competition ([1], p. 16). Collaboration may also include the "mere" coincidence of individual interest, and it is often in the interest of any individual to collaborate at least to some extent ([1], p. 2).

\subsection{Lineage Formation}

Life, according to the essay, occurs at the intersection of lineage formation and collaborative involvement in metabolism ([1], p. 14). If life is viewed in this way as the result of the intersection of lineage-forming, metabolically collaborative matter organized within different interacting levels, then the smooth transition from the earliest living matter to standard examples of life and beyond all the way up to contemporary ecosystems should be possible ([1], p. 16).

\subsection{Living}

The essay does not aim to provide an answer to Schrödinger's question “What is Life" [3]. Rather the intent is to present a spectrum of biological entities that illustrates why no sharp dividing line between living and non-living things is likely to be useful. Instead, the goal is to offer a view of life that explains why organizations of matter can be described as living ([1], pp. 1-2). A necessary condition for being a living thing is the ability to reproduce, however, it is not obvious that this is sufficient. Living entities must also have the capacity to sustain themselves through biochemical transformations. On that account metabolism can be engaged in autonomously or cooperatively, through interaction with other biological entities ([1], p. 3).

\subsection{Metabolic Whole}

Life can be seen as something that arises only at the intersection of two features: matter is living when lineages are involved-directly or indirectly-in metabolic processes. The difficulty that arises in the attempt to comprehend life is the observation that the entities which form lineages are not always the same as those that form metabolic wholes. Consequently, it can be argued that metabolism, the transformative biological reactions that sustain life processes, is a collaborative affair. Hence the claim that life is typically found at the collaborative intersection of many lineages, together with the suggestion that collaboration should be seen as a central characteristic of living matter, is also a claim that has implications for how we understand the origins of life ([1], p. 2).

\subsection{Non-Living}

Many biologists deny that viruses are living organisms, yet viruses are frequently considered to be test cases for the boundary between life and non-life, organism and non-organism, and biology and chemistry, whereby they are most often relegated to the second of each of these category pairs. In that light, viruses are re- 
garded as non-living on the grounds that they cannot reproduce themselves autonomously, nor can they metabolize. They can, nevertheless, carry out such biologically impressive activities as entering cells to gain access to the transcription and translation machinery of the cell for their own use. Thus, they are able to move about the DNA from the organism with which they interact. Hence, by exploiting or collaborating with cellular organisms in these ways, they very effectively reproduce themselves and have no requirement of autonomous metabolism [1].

Recalling what was said at the end of (2), namely, that (3) will reveal how things relate to evolution, and (b) how a microorganism can use ATP without being able to produce it, the above shows that the Dupré \& O'Malley essay [1] extensively answers such questions within the context of the suggested keywords. However, the above also shows that a very important keyword or term, namely ATP, must be added to the list of keywords to achieve some clarity about what role in metabolism, and in particular in collaborative metabolism or metabolic processes, ATP plays in biology.

This is also of particular importance with regard to the status of viruses. They can only keep reproducing for a prolonged period of time, as Schrödinger has it [3], if they have access to energy, which only ATP can provide for them. Moreover, including ATP as a major key-term emphasizes the importance of the distinction between (a) having a metabolism and (b) partaking in metabolic processes, rather than de-emphasizing this.

Since processes involving the adenosine phosphates AMP, ADP, and ATP are central to metabolism, it can be concluded that viruses rely on this for their external source for energy more so than any other entities considered in this review. In short, ATP is food for viruses. It is the energy of ATP that sustains their activities and keeps them doing what they were meant to do, which is to reproduce and thus to survive. And for that, viruses do not depend on what we believe or think with regard to whether they are alive or not.

\section{Conclusions}

With regard to the essay's central argument that life arises when lineage forming entities collaborate in metabolism, it seemed that we needed a more explicit description of what metabolism in biology entails. By taking a close look at the three fundamental questions (1), (2), and (3) and by taking (a), and (b) into consideration, it can be shown that if the role of ATP is also accounted for, then a more straightforward description of metabolism in biology can be produced. The inclusion of ATP in this discussion does not diminish the essay's central argument, but rather strengthens it by providing the missing link between the central argument and the three fundamental questions.

Moreover, by adding ATP to the discussion, the important role that viruses play in biology also becomes more explicit. Furthermore, if one views ATP as the perfect energy currency for the cell, then that currency can best be explained 
with regard to physics. It is not difficult to calculate that the energy liberated by the conversion of 1 mole of ATP to the energy of one molecule of ATP amounts to $6.64 \times 10^{-20}$ joules, which can be related to the standard unit definition of $1 \mathrm{eV}$ (electron volt) which is equivalent to $1.60 \times 10^{-19}$ joules of energy.

One may assume that these infinitesimally small amounts of energy are insignificant but, when one considers that a virus must deal with its needs for energy to sustain its activities by picking up each infinitesimally small quantum of energy one at a time, the provision of energy by a single ATP molecule is not insignificant, given that only two and a half conversions from ATP to ADP are required to generate $1 \mathrm{eV}$ of energy. And, of course, the electron volt plays an important role in measuring electron transport. Further information of how ATP levels of living cells can be visualized and measured can be found in [7]. In short, by looking at things in terms of biophysics, it would appear that there is no longer any reason for viruses to be placed separately in the gray area of the nonliving, given that they use the energy provided by the ATP molecules of living cells in order to reproduce and survive. Thus, viruses associate in symbiosis with living cells. Consequently, it can be concluded that to reproduce and survive, viruses developed the ability to interact in symbiosis with living cells to take advantage of their ATP energy production mechanism in a non-collaborative parasitic manner that often leads to the death of the cells but, this also ensures the survival of the viruses' lineages.

\section{Acknowledgements}

I want to express my gratitude to Dr. Jerry Bergman and my colleague André Michaud for the help they provided by reviewing my manuscript.

\section{Conflicts of Interest}

The author declares no conflicts of interest regarding the publication of this paper.

\section{References}

[1] Dupré, J. and O’Malley, M.A. (2009) Varieties of Living Things: Life at the intersection of Lineage and Metabolism, Philosophy and Theory in Biology. University of Exeter, Exeter. http://dx.doi.org/10.3998/ptb.6959004.0001.003 https://quod.lib.umich.edu/cgi/t/text/text-idx? $\mathrm{cc}=\mathrm{ptb} ; \mathrm{c}=\mathrm{ptb} ; \mathrm{c}=\mathrm{ptpbio} ; \mathrm{idno}=6959004$ $.0001 .003 ;$ rgn $=$ main; $v i$ w $=$ text; $\mathrm{xc}=1 ; \mathrm{g}=$ ptpbiog

[2] Maruyama, K. (1991) The Discovery of Adenosine Triphosphate and the Establishment of Its Structure. Journal of the History of Biology, 24, 145-154.

[3] Schrödinger, E. (1944) What Is Life? The Physical Aspect of the Living Cell. Cambridge University Press, Cambridge. http://www.whatislife.ie/downloads/What-is-Life.pdf

[4] Bergman, J. (1999) ATP: The Perfect Energy Currency for the Cell. Creative Research Society Quarterly, 36. https://www.trueorigin.org/atp.php

[5] Hui, E.K.-W. and Nayak, D.P. (2002) Role of G Protein and Kinase Signaling in In- 
fluenza Virus Budding in MDCK Cells. Journal of General Virology, 83, 3055-3066. https://doi.org/10.1099/0022-1317-83-12-3055 https://pubmed.ncbi.nlm.nih.gov/12466482/

[6] Zaitlin, M. (1998) The Discovery of the Causal Agent of the Tobacco Mosaic Disease. In: Kung, D. and Yang, S.F., Eds., Discoveries in Plant Biology, World Publishing Co., Ltd., Hong Kong, 105-110.

https://doi.org/10.1142/9789812817563 0007

https://www.apsnet.org/edcenter/apsnetfeatures/Pages/DiscoveryCausalAgentTobac coMosaicVirusDisease.aspx

[7] Ando, T., et al. (2012) Visualization and Measurement of ATP Levels in Living Cells Replicating Hepatitis C Virus Genome RNA. PLoS Pathogens, 8, e1002561.

https://doi.org/10.1371/journal.ppat.1002561

https://pubmed.ncbi.nlm.nih.gov/22396648/ 